\title{
CONTINUIDADES Y RUPTURAS ENTRE FORMACIÓN INICIAL Y EJERCICIO PROFESIONAL DOCENTE ${ }^{1}$
}

Marisol Latorre Navarro

Doctora en Educación. CIDE. Universidad Alberto Hurtado

\section{LOS INICIOS DE LA REFORMA Y EL ESTADO ACTUAL DE LA CUESTIÓN}

1. Desde sus inicios en 1990, la reforma a la educación en Chile asumió a la educación como vector estratégico para el desarrollo del país en sus distintas dimensiones: crecimiento económico, superación de la pobreza, consolidación de un régimen democrático, formación de las nuevas generaciones en valores cívicos y ciudadanos (García Huidobro, 1999). Calidad y equidad del sistema escolar fueron definidos como los núcleos orientadores de los mejoramientos y cambios a lograr.

Las autoridades han concentrado, particularmente, su atención y los esfuerzos en la dimensión pedagógica, lo que se ha traducido en la formulación de diversas propuestas para avanzar en el mejoramiento (en un primer momento) y la transformación (posteriormente) de las prácticas de enseñanza y aprendizaje de maestros y alumnos en las escuelas del país.

2. Resultados obtenidos en evaluaciones recientes, tanto nacionales como internacionales, han atraído el interés de investigadores, académicos, profesores, estudiantes y de la opinión pública en general, particularmente porque estos resultados aportan evidencia de que, si bien, en la última década se ha logrado mejorar la educación del país, dicha mejora parece insuficiente frente a las expectativas existentes.

Una de las instancias que mide el logro de aprendizajes, en distintos niveles de enseñanza y a lo largo de todo el país, es la evaluación SIMCE (Sistema Nacional de Medición de la Calidad de la Educación). Se trata de una evaluación que se realiza todos los años, que se aplica en un nivel cada vez alternando entre 4ํ , 8ำ años de Educación General Básica o Primaria o $2^{\circ}$ años de Enseñanza Media o Secundaria $^{2}$ y que considera las áreas de Lenguaje y comunicación, Matemáticas y Comprensión del Medio (ciencias).

Los resultados arrojados por la evaluación realizada en el año 2002 a $4^{\circ}$ años básicos, evidencian que en dos de las tres áreas evaluadas los estudiantes lograron acceder al promedio de 250 puntos establecido para cada una de las pruebas. En esta situación se encuentran Lenguaje y Comunicación y Comprensión del Medio, pero en situación distinta está el área de Matemáticas, donde los estudiantes estuvieron bajo el promedio con 247 puntos (www.simce.cl).

\footnotetext{
${ }^{1}$ Este artículo ha sido elaborado en el marco de la investigación: Prácticas pedagógicas y formación de profesores: desafíos pendientes. Aportes para el mejoramiento de la calidad y equidad de la educación chilena (2003-2005), Proyecto Fondecyt Postdoctoral 3030013.

${ }^{2}$ Al respecto hay que señalar que la estructura del sistema educacional chileno se articula en base a la existencia de 2 niveles fundamentales: Enseñanza Básica (organizada en 8 niveles de educación, que van desde $1^{\circ}$ a $8^{\circ}$ años de enseñanza básica) y Enseñanza Media (organizada en 4 niveles de educación, que van desde $1^{\circ}$ a $4^{\circ}$ años de enseñanza media).
} 
Si se comparan los resultados obtenidos en el año 1999, anterior oportunidad en la que el SIMCE evaluó el mismo nivel de enseñanza, con los del año 2002, se advierte que en términos de resultados escolares no ha habido avances significativos ${ }^{3}$.

Al observar los resultados obtenidos en el SIMCE año 2002 distribuidos en los distintos grupos socioeconómicos a los que pertenecen los estudiantes evaluados, se ve que existen importantes diferencias entre los logros que alcanzan los alumnos de los distintos grupos. Un cálculo del promedio entre los tres sectores para cada uno de los grupos arroja que existen 77 puntos de diferencia entre los rendimientos de los alumnos del grupo bajo y los del grupo alto ${ }^{4}$. Claramente, mientras más alto es el nivel socio-económico de origen de los estudiantes, mejores son los resultados de rendimiento que obtienen.

A fines del año 2004 fueron dados a conocer los resultados obtenidos por Chile en el TIMSS 2003 (Third International Mathematics and Science Study) y que son, por cierto, bastante desalentadores: Chile se sitúa en el lugar 39 entre 46 países, aunque es el único país latinoamericano en rendir la prueba; si se comparan los rendimientos obtenidos por los alumnos chilenos en la misma prueba el año 1999 y el año 2003, se refleja un descenso en matemática de 392 a 387 y en ciencias, de 420 a 413 puntos. Lo anterior implica que de entre los casi cincuenta países participantes en el estudio, Chile se ubicó entre los diez con peor rendimiento en estas dos asignaturas

Los alumnos chilenos que participaron en TIMSS 2003 fueron de los primeros en estudiar con los nuevos programas de estudio, introducidos por la reforma educacional. Si se compara el nuevo currículo con el anterior, este posee una definición más específica respecto de los contenidos y está más en línea con los estándares internacionales de enseñanza, enfatizando más la resolución de problemas en contextos cotidianos, la aplicación de conceptos y el razonamiento científico.

Sin embargo, a pesar de estos avances, el nuevo currículo no ha mejorado el rendimiento. Esto se relaciona con el hecho de que el nuevo currículo no se ha traducido en cambios sustantivos en las prácticas docentes. Un buen currículo es condición necesaria, pero no suficiente, para una educación de calidad. En Chile, al igual que hace cuatro años, el énfasis de las clases de matemáticas sigue puesto en el aprendizaje de conceptos numéricos básicos, como fracciones y decimales, con lo cual los alumnos tienen menos oportunidades para aprender matemáticas avanzadas, comparado con los alumnos de otros países que tienen mejor rendimiento.

\section{II. ¿CÓMO ES POSIBLE EXPLICAR TALES RESULTADOS?}

3. Los análisis al respecto dan cuenta de la presencia e influencia que ejercen dos conjuntos de variables: de una parte, aquellas relacionadas con el nivel socio-económico y cultural de pertenencia de los estudiantes y, de otra, aquellas que dicen relación de las características de las escuelas a las que estos estudiantes asisten.

Según Schiefelbein (2004):

\footnotetext{
3 "La medición arrojó como resultado promedios totales nacionales de 251 puntos para Lenguaje y Comunicación, 247 puntos para Matemática y 251 puntos para Comprensión del Medio (Ciencias Sociales y Naturales), lo que implica que no hubo variaciones significativas de los resultados en relación al año 1999" (www.simce.cl).

${ }^{4}$ Para ver más, visitar sitio www.simce.cl
} 
"El Simce 2003 muestra nuevamente que las escuelas no compensan las diferencias de recursos en el hogar. El aprendizaje de los niños está asociado al nivel socioeconómico de la familia".

Esta situación pone en tensión la relación escuela-familia, pues si bien el conjunto de variables que dicen relación con las familias y el entorno familiar de los alumnos del sistema escolar inciden significativamente en los aprendizajes que sus hijos pueden lograr, se trata de variables que pertenecen a un ámbito 'extra-escuela', que incluye elementos 'no alterables' para la escuela, es decir, elementos de los cuales la escuela no puede hacerse cargo ni modificar directamente. En este sentido, el nivel de escolaridad de los padres, el apoyo y acompañamiento que estos puedan brindar a sus hijos en sus estudios, así como la comunidad o barrio donde está inserta la escuela son algunos de los aspectos importantes a considerar.

Pero existe otro conjunto de variables que pueden ser consideradas 'alterables' o directamente modificables por cada escuela; se trata de variables 'intra-escuela', variables que requieren ser identificadas y trabajadas con el objetivo de potenciar su aporte como 'valor agregado' en función de los aprendizajes esperados por la escuela respecto de sus alumnos. En este contexto, una de las variables alterables de la cual la escuela puede y debe responsabilizarse es la que refiere a las prácticas pedagógicas y, más específicamente, a las prácticas de enseñanza que llevan a cabo los docentes en las aulas de las escuelas del país.

Respecto a la identificación de estos dos conjuntos de variables, resulta pertinente la afirmación hecha por Reimers:

"la buena enseñanza explica un porcentaje equivalente de la variación en los desempeños académicos de los estudiantes a la explicada por el nivel educativo de los padres y por condiciones socioeconómicas" (Reimers, 2003:42).

Es ineludible que la escuela se haga responsable de saber cuáles son las variables que influyen más en su propia realidad, identificarlas, describirlas, ponderarlas, analizarlas y decidir cómo trabajar con ellas para lograr mejores resultados con los alumnos que tiene. Para lograr esto la escuela requiere ser capaz de asumir y gestionar, de la mejor manera posible, su proyecto educativo y su capital simbólico de sentido y motivación común (Bellëi y otros, 2004:51), es decir, aquello que la guía y orienta, su visión, ideario y misión, sus objetivos, normas y reglamentos, ... pero para hacerlo requiere de la articulación entre herramientas conceptuales sólidas y pertinentes y de experiencias prácticas exitosas. Esta es una tarea que reviste mayor importancia, aun, cuando se trata de escuela que atienden a los alumnos de niveles socioeconómicos desfavorecidos.

4. Al interior del contexto escolar, profesores y profesoras se han constituido en quizá la principal "punta de lanza" de los procesos de mejoramiento y transformación conducidos por las autoridades. Sobre ellos recaen muchas miradas que intentan desentrañar con la mayor exactitud posible qué hacen los profesores, cómo lo hacen y cómo sus prácticas se relacionan con los desafíos que le plantea la sociedad a la escuela y las demandas específicas de cambio que le formulan las autoridades. Los rendimientos académicos de los alumnos son, en la actualidad, un referente privilegiado a la hora de caracterizar y, sobretodo, evaluar las prácticas que realizan los profesores y sus efectos.

A partir del segundo semestre del año 2003, se implementó en el país el Sistema de Evaluación del Desempeño Profesional Docente. Este sistema de evaluación fue aplicado a 3.700 profesoras y profesores 
de establecimientos municipales, un tipo de escuelas que constituyen mayoría en el país, quienes fueron evaluados voluntariamente.

Los resultados muestran que, desde la perspectiva de su desempeño: el $4 \%$ de los docentes evaluados se ubican en la categoría "insatisfactorio", el 30\% se ubica en la categoría "básico", más de la mitad (57\%) se sitúa en la categoría "competente", y quienes se desempeñan de manera "sobresaliente" representan el $9 \%$ del total (Mineduc, 2003).

En otras palabras, aproximadamente, un tercio de los profesores y profesoras evaluados presentan desempeños profesionales acordes con las características de los niveles inferiores, frente a lo cual requieren ser apoyados para mejorar. Mientras que dos tercios de los profesores y profesoras pueden ser ubicados en las dos categorías superiores. Es decir, gran parte de los profesores evaluados poseen los conocimientos y las habilidades 'adecuados' para responder a las necesidades y demandas actuales de la escuela y, por lo tanto, es posible hipotetizar que estarían llevando a cabo prácticas de enseñanza que podríamos identificar en términos generales como 'buenas' de acuerdo a los criterios y patrones establecidos por los evaluadores.

Los resultados obtenidos pueden parecer tranquilizadores, pero sin embargo no aportan elementos que permitan clarificar ni comprender por qué niños y niñas no logran aprender aquello que el sistema estima y define como necesario y apropiado para cada nivel de enseñanza?. Un ejemplo de ello es que aproximadamente un $50 \%$ de los estudiantes chilenos de $3^{\circ}$ y $4^{0}$ años de educación general básća (primaria) no entienden las explicaciones que sus profesores y profesoras les dan en clases.

Algunos de los principales hallazgos presentados por el informe de la OECD (2004) plantean una actitud de preocupación ante las limitadas conexiones entre la formación de los docentes, su desarrollo profesional y las necesidades de las escuelas, la mantención de la oferta de profesores de buena calidad (particularmente en materias de alta demanda), las características de la composición de la 'fuerza docente' (menos 'altamente competentes', menos jóvenes y menos varones), las altas tasas de deserción especialmente entre los nuevos docentes y la inequitativa distribución de docentes de calidad entre los distintos tipos de escuelas.

\section{LA IMPORTANCIA DE CONSIDERAR LAS PRÁCTICAS PEDAGÓGICAS DE LOS MAESTROS}

5. Evidencia de investigaciones recientes han señalado que los buenos maestros marcan una clara diferencia en los aprendizajes que logran sus alumnos, en sus rendimientos y, en definitiva, en el éxito escolar que estos alumnos puedan tener (Rivkin y otros, 2002; Reimers, 2003), que los docentes varían sustancialmente en su efectividad (entre docentes, un mismo docente a lo largo de su vida y un mismo docente en distintas escuelas) y que las diferencias en los aprendizajes de los estudiantes son frecuentemente mayores dentro de una misma escuela que entre escuelas distintas (OECD, 2004).

En un contexto educacional caracterizado por la heterogeneidad, subsisten las enormes esperanzas de que estudiantes latinoamericanos, y chilenos por cierto, y sus familias, poseen en sus maestras y en la educación escolar, la alta valorización social que posee la institución escolar, particularmente en Chile y México, frente a lo poco que los estudiantes de estos mismos países logran aprender en la escuela, particularmente aquellos que provienen de sectores pobres (Reimers, 2003:33-34). 
En medio de este escenario, las prácticas pedagógicas de profesores y profesoras en ejercicio se evidencian como ineludible núcleo de interés, pero al mismo tiempo de tensión, en donde se encuentra contenido un potencial de saber y cambio estratégicos.

Para el caso de Chile, basándose en resultados de investigaciones, es posible señalar que entre las principales características que presentan las actuales pácticas de enseñanza de los docentes están las siguientes:

- Las prácticas pedagógicas se sustentan en creencias. Estas creencias son previas al ingreso que hacen quienes quieren ser profesores, a los programas de formación inicial y difícilmente dichos programas las modifican. Los profesores creen que la mejor forma de enseñar es la que se realiza de acuerdo a parámetros tradicionales, es decir, sobre la base de establecer relaciones verticales y poco interactivas entre profesor y alumnos (González, 1999).

- La primera experiencia profesional ejerce un efecto importantísimo en las prácticas futuras. Al momento de iniciarse profesionalmente, quienes tuvieron una mala experiencia señalan como objetivo prioritario aspectos de orden formativo, mientras que quienes tuvieron una experiencia exitosa, se focalizan en aspectos de orden intelectual (Pasmanik, 2001).

- En las prácticas predominan saberes de sentido común y de la experiencia. Respecto de los saberes que poseen y utilizan los profesores en sus prácticas de enseñanza, existe un predominio de saberes de sentido común y saberes de la experiencia, adquiridos en su propia experiencia escolar (como alumnos de escuelas primarias y secundarias) y en el contexto profesional en el cual están insertos (pares, cultura escolar), lo que favorece la reproducción de estrategias y metodologías de enseñanza aprendidas y replicadas de manera poco crítica (Latorre, 2002).

- Los contenidos disciplinares son dominio del profesor. La relación entre profesores y alumnos se desarrolla, privilegiadamente, a través de los contenidos de asignatura y estos son dominio del profesor (Villalta, 2000). Sin embargo, los profesores no enseñan la totalidad de los contenidos que definen los programas de estudio para cada nivel y período escolar ni utilizan la totalidad del tiempo disponible para enseñar. Al interior de la hora pedagógica (de 45 minutos) los tiempos de enseñanza ocupan, aproximadamente, un tercio, es decir, 15 minutos y el resto del tiempo se distribuye en pasar la lista de los alumnos, controlar el orden y la disciplina para dar inicio a la hora de clases y dar instrucciones.

- La estructura de la clases se basa en un diseño lineal. En la realidad intra-aula predomina un trabajo construido centralmente sobre un diseño lineal, los docentes son bastante reacios a incorporar cambios estructurales importantes en sus planificaciones previas y su quehacer. Los aportes de los estudiantes son considerados solamente durante la clase misma, pero los docentes no los incorporan como elementos nuevos a su ejercicio profesional. El énfasis de la clase está en la concreción reproductiva de la estructura planificada previamente, lo que evidencia una gestión pedagógica instaurada a partir de la normatividad (Guzmán, 2002).

- Hay debilidades y vacíos en los contenidos a enseñar. Un aspecto en el que hay coincidencia entre lo señalado por el informe de la OCDE (2004) y los resultados de Chile en el 
TIMSS, es que los docentes chilenos, actualmente en ejercicio, poseen debilidades importantes respecto del conocimiento y dominio de los contenidos disciplinares que deben enseñar a sus alumnos, de las estrategias didácticas específicas para hacerlo y de las relaciones que se pueden establecer entre los contenidos de su asignatura y otros campos del saber.

\section{IV. ¿CÓMO Y DÓNDE APRENDEN PROFESORES Y PROFESORAS A SERLO?}

6. Esta pregunta parece relevante a la luz de los antecedentes presentados, pues parece esencial saber cómo llegaron los profesores en ejercicio a poseer prácticas de tales características; una pregunta que evidencia la tensión existente entre la preocupación que hoy existe frente a la calidad de la educación y la calidad de las prácticas pedagógicas que realizan profesores y profesoras.

Se trata de una inquietud 'razonable' si reconocemos que las prácticas docentes producen efectos tanto en los aprendizajes y resultados académicos de los alumnos como en la totalidad de los aprendizajes que tales alumnos pueden lograr y en su personalidad. A esto se puede agregar que, aun más, el efecto de la práctica pedagógica de un maestro no es una influencia aislada, sino que se adiciona y se combina con el efecto de las prácticas pedagógicas de los demás profesores que tiene ese alumno y con los efectos producidos por las prácticas de gestión institucional que tienen lugar en la escuela a la que el alumno asiste.

En otras palabras, es posible plantear que los resultados educativos que logra un estudiante son producto de la agregación de los efectos del conjunto de las prácticas escolares en las cuales dicho estudiante está inserto. Situación que se ve fortalecida con implementación de la Jornada Escolar Completa (JEC) donde los tiempos de permanencia de profesores y alumnos en las escuelas se extienden y aumentan las instancias de interacción entre profesores y alumnos y el impacto de los efectos que las prácticas escolares tienen sobre niños, niñas, maestros y maestras en la escuela y sobre la comunidad escolar en su conjunto.

De ahí la importancia de las prácticas pedagógicas en la escuela y la necesidad de considerarlas como un objeto-espacio de atención prioritaria, tanto desde la perspectiva de los profesores, como de los equipos directivos, académicos, investigadores y autoridades políticas en educación.

7. En la búsqueda de respuestas posibles a la pregunta formulada, la más obvia es la que remite al campo de la formación docente y, más específicamente, a la formación inicial docente.

A este respecto, en su tesis doctoral Fuentealba (2003) concluye que:

"La Formación inicial recibida por los (profesores) Principiantes sería uno de los elementos que explicaría la calidad del desempeño profesional" (2003:p.191)

Sin embargo, no hay evidencia empírica que dé cuenta de qué elementos y aprendizajes adquieren los profesores en su formación inicial y cuáles utilizan en su práctica los profesores que de ella egresan y se titulan.

Más bien, hay evidencia en el sentido inverso. En el 2001, el informe de IIPE-UNESCO, señala el bajo impacto de la formación inicial, como instancia de preparación, sobre las prácticas pedagógicas de profesores principiantes, agregando que quienes se inician profesionalmente, a la hora de "hacer clases", tienden a refugiarse en sus imágenes previas (de los profesores que tuvieron ellos mismos siendo alumnos en la escuela) o se someten inmediatamente a las demandas del contexto escolar específico en el que 
deben trabajar (Vaillant, 2002); es decir, 'aprenden' en su temprana experiencia docente, lo que hace suponer que 'des-aprenden' aquello adquirido en la formación inicial o 're-aprenden' experiencialmente, a través de su propia práctica como docentes y la de otros colegas, a ser profesores.

Frente a la pregunta por su formación inicial, profesores y profesoras en ejercicio, se posicionan de una manera bastante crítica. Ellos y ellas señalan, en términos generales, que aprendieron poco y que no fue de utilidad a la hora de comenzar a realizar sus prácticas profesionales, tanto dentro como fuera del aula (Latorre, 2002), más aun critican la carencia de mística y la poca preocupación de los formadores de formadores por 'seducir en la profesión' (Fuentealba, 2003:191). Ambas investigaciones, además, coinciden en que tales críticas se dirigen, principalmente, a la formación inicial pedagógica más que a la formación disciplinar.

Estos antecedentes permiten a lo menos discutir el supuesto de que existe, o podría existir, una relación lineal y directa entre el campo de la formación docente y el campo del ejercicio profesional docente.

La puesta en discusión de este supuesto lleva a formularnos interrogantes en dos sentidos: primeramente, ¿Ejerce la formación inicial un impacto significativo en las futuras prácticas pedagógicas de los futuros profesores? y la segunda, ¿Las prácticas pedagógicas que hoy realizan profesores y profesoras en el contexto escolar están sustentadas en los aprendizajes adquiridos en espacios e instancias formales de formación?

El informe de la OECD (2004) atribuye a la formación inicial de profesores parte importante de la responsabilidad en los resultados educativos, Ilamando la atención hacia a existencia de debilidades, principalmente en el dominio de la materia, las relaciones entre los contenidos disciplinares y las metodologías que los profesores utilizan en la sala de clases.

Aparentemente, existe una tensión entre aquello que los formadores de formadores enseñan, aquello que los futuros profesores, aparentemente, aprenden y aquello que, posteriormente, ponen en juego y hacen cuando se desempeñan profesionalmente. Si los profesores no adquieren en la formación inicial elementos significativos que les sean útiles para desempeñarse como tales, el riesgo latente es el vacío de no saber qué ni cómo hacer y la natural reproducción de las acciones existentes en su entorno pasado o presente (hacer lo mismo que otro hacía o hace). De ser así, las posibilidades de cambio se minimizan, desaparecen o quedan sujetas a la emergencia de ideas e iniciativas espontáneas y respuestas frente a situaciones coyunturales y específicas.

El campo de la formación inicial es un campo que requiere ser investigado, no solo en sus características más estructurales y generales, sino también en sus aspectos relacionales, es decir, en los vínculos e interrelaciones que establece internamente entre sus componentes y también, con el exterior, particularmente con aquellos docentes a los cuales ha formado y que hoy se desempeñan en los distintos ámbitos y niveles del sistema escolar.

8. Retomando la pregunta formulada en este apartado ¿Dónde y cómo aprenden los profesores a serlo? La propia escuela, aquella donde profesores y profesoras realizan sus prácticas pedagógicas cotidianamente, también emerge como un campo formativo posible.

El que los profesores tiendan a repetir los modos de enseñanza de sus profesores en la escuela y/o los de sus pares de la escuela en la que se desempeñan, es una situación que, según algunos autores, 
puede ser explicada si se considera que cada unidad educativa, en tanto organización que posee una cultura escolar propia y específica, acoge y, a la vez, obliga a sus docentes a insertarse (y adaptarse) a rutinas preestablecidas. Sin embargo, junto a tales rutinas, existen también espacios no rutinizados que encierran un potencial de cambio posible. En cada escuela coexisten espacios y acciones que están reglados y tienden a la mantención del orden y la cultura escolar vigente junto a espacios y acciones noreglados donde es posible encontrar acciones tendientes a generar nuevas maneras de ser, aprender y formar (Tickle, 2000, Garant, 1999).

El contexto-campo de la escuela ejerce una influencia, que no puede ser desconocida ni minimizada, sobre los profesores y profesoras en ejercicio y, en consecuencia sobre las características de las prácticas pedagógicas que estos realizan. Si consideramos la influencia que aparentemente ejerce la formación inicial frente a aquella que parece tener el 'efecto escuela' en las prácticas docentes, es posible pensar que las acciones que los docentes llevan a cabo están fundadas en razones y argumentos que tienen sentido para ellos al interior de su escuela y no, tan claramente, fuera de ella y ante los ojos de otros. Motivaciones, creencias y razones que los profesores, a lo menos concientemente, no asocian con su formación inicial.

9. Parece pertinente avanzar en identificar y analizar frente a frente los aportes provenientes tanto del campo de la formación inicial como del campo del ejercicio profesional docente, desde la perspectiva de los profesores como actores relevantes en los procesos de enseñanza y aprendizaje escolar. ¿Es que acaso, las 'buenas razones' (o lo que algunos autores llaman 'racionalidades') del campo de la formación inicial son distintas de las 'buenas razones' del campo escolar? ... para contestar esta pregunta, es necesario previamente avanzar en identificar ¿Cuáles son las 'buenas razones', argumentos, sentidos, racionalidades construidas y vigentes en uno y otro campo?, ¿Cuáles y cómo son en la actualidad las relaciones entre las 'buenas razones' de estos dos campos?

La tensión es latente ... ¿Qué características tienen las 'buenas razones' que subyacen a las prácticas pedagógicas de profesores y profesoras en la escuela?, ¿Cómo y dónde las construyeron? y ¿Cómo es posible trabajar con ellas, desde una perspectiva de formación?

\section{EL APORTE DEL ESTUDIO DE LAS RACIONALIDADES}

10. Tradicionalmente las prácticas pedagógicas han sido concebidas como las acciones o actividades que realizan profesores y profesoras al interior de las salas de clases. Sin embargo, no cualquier acción que realiza un profesor en una aula es pedagógica ni educativa. Ampliar la conceptualización tradicional implica entender que las prácticas pedagógicas son un 'continuum de acciones dotadas de sentido', y más aun, no de cualquiera, sino de un cierto sentido de significación específica que posee validez y legitimidad ante los ojos del actor que las lleva a cabo.

Cada uno de los profesores y profesoras que hoy lleva a cabo una práctica pedagógica de determinadas características, la realiza de tal manera, porque tiene 'buenas razones' para hacerlo de ese modo y no de otro. Estas 'buenas razones' están relacionadas con un cierto conjunto de creencias, saberes y conocimientos que el profesor posee y, desde el cual, construye individualmente su propio 'sentido' de significar y hacer las cosas. Un sentido que el profesor actualiza en las acciones que constituyen su práctica profesional. 
11. La investigación en curso se inserta en una línea de comprensión de la práctica pedagógica entendida como el resultado de acciones, decisiones, actitudes, creencias y comportamientos individuales; elementos que se articulan entre sí para producir 'buenas razones', es decir, motivaciones y mecanismos por los cuales se dan puntos de apoyo a partir de los que objetivos y modalidades de la acción pueden ser legitimados a los ojos de los actores sociales; y que, dichas 'buenas razones' constituyen las bases para que los actores piensen lo que piensan, crean lo que creen y hagan lo que hacen (Boudon, 2003).

Las 'buenas razones' no tienen que ser necesariamente éticas ni moralmente verdaderas o correctas, basta con que sean 'buenas' para el actor y legitimadoras de la acción que este realiza. Es por esta característica, propia de su naturaleza, que dichas razones no se construyen ni pueden ser comprendidas a distancia del individuo que las sustenta, por eso es imprescindible retomar, conocer y comprender (pero no necesariamente compartir o validar) la perspectiva del individuo que detenta tal o cual razón y, en virtud de ella, realiza tal o cual acción.

Desde la perspectiva del estudio de las racionalidades, enfoque teórico en el cual se inscribe esta investigación, se reconoce el carácter del individuo como 'actor social situado', es decir:

- Como entidad pensante, que ejerce su libertad y que es capaz de reconocer y asumir, individualmente, sus responsabilidades ante sí mismo y los demás

- Como individuo condicionado por el sistema de interacciones y la estructura social en que está inmerso y que, al interior de dicho sistema y dicha estructura, participa de relaciones de interacción social (individuo-individuo o actor-actor; actor-contexto) y asume cierta posición (en términos de saber y poder).

Esta mirada teórica implica, para la educación como nuestro campo de estudio, el reconocimiento de profesores y profesoras como actores pedagógicos insertos en un sistema y en un contexto socioprofesional específico (el sistema escolar y la escuela) al interior del cual actúan, con un cierto margen de autonomía de decisión, según sus propias motivaciones y decisiones, reflexionando, sopesando y deliberando sobre los acontecimientos y la realidad que los rodea. Desde esta perspectiva se hace posible acceder a conocer y develar ¿Cuáles son las 'buenas razones' por las cuales los profesores realizan sus prácticas profesionales de la manera en que lo hacen?, ¿Cuáles son sus motivaciones?, ¿Dónde y cómo las construyeron?

En síntesis, las prácticas pedagógicas son un objeto de atención significativo si se quiere modificar y mejorar los resultados de la escuela, pero para avanzar en ese sentido es necesario indagar tanto en las acciones que realizan los profesores y sus debilidades como en los supuestos que dichos profesores tienen y ponen en juego al momento de actuar.

\section{DESAFÍOS PENDIENTES ...}

12. Falta más investigación sobre el tema. Hasta ahora se ha asumido casi naturalmente que la formación inicial docente logra impactar y transferir conocimientos y saberes acumulados en distintos campos del conocimiento a los futuros profesores y que, al término de un proceso formativo -de corta duración (1 ó 2 años) o más largo (4 ó 5 años)-, el futuro profesor o profesora ya estará 'formado' en determinadas dimensiones (conocimientos, saberes, habilidades) y en condición de ser 'certificado', 
'diplomado' o 'titulado' como profesional especialista en el área. Así, casi por añadidura, todo profesor y toda profesora estará en posesión de las competencias necesarias para llevar a cabo buenas prácticas pedagógicas que favorezcan una buena enseñanza y promuevan aprendizajes de calidad en sus alumnos.

La pista que, desde esta investigación seguimos es el actor situado, el profesor o profesora que habiendo participado de procesos e instancias de formación inicial, hoy enseña en las aulas de las escuelas de nuestro país con resultados diversos. Desde esta perspectiva, el reconocimiento de la 'posicionalidad' del actor social reviste un carácter doble: como actor situado en el campo de la formación inicial y, a la vez, en el campo del ejercicio profesional.

Son las 'buenas razones' presentes, tanto en el campo de la formación inicial como en el campo de la escuela y sus relaciones, lo que nos interesa develar. Ellas constituyen el objeto de estudio de esta investigación. Para acceder a dicho objeto, se hace necesario conocer, describir y caracterizar las prácticas pedagógicas de profesores en ejercicio y recuperar la perspectiva del profesor en ejercicio en tanto sujeto formado por un programa de formación inicial caracterizado por determinadas 'buenas razones' y sujeto inserto en un contexto escolar caracterizado, también, por ciertas 'buenas razones'. ¿Son las 'buenas razones' de uno y otro campo concordantes entre sí?, ¿Son discordantes? o ¿Coexisten aspectos de concordancia y discordancia?

Resultados preliminares de la investigación muestran que al interior del grupo de profesores chilenos considerados en el estudio, se pueden identificar dos sub-grupos que se diferencian por las expectativas que tienen respecto de los aprendizajes que sus estudiantes pueden lograr y de los objetivos que se plantean respecto de sus prácticas de enseñanza. Esta distinción no es coincidente con los tipos de instituciones donde se formaron inicialmente estos profesores y profesoras, tampoco con los tipos de administración que rigen a las escuelas en donde trabajan.

Donde sí aparecen asociaciones es con los resultados de aprendizaje que obtienen los estudiantes de estas escuelas, es decir, escuelas que obtienen mejores resultados difieren de las que no los logran en las expectativas que los profesores tienen de sus estudiantes y en los objetivos de aprendizaje que se plantean.

La evidencia es sugerente en el sentido de que estaría dando cuenta del peso que ejerce el "efecto escuela" sobre sus profesores y sus prácticas, tensionando el impacto del efecto de las características de la formación inicial.

La constatación empírica de que existe un distanciamiento entre los campos es un primer hallazgo útil que sugiere la necesidad de seguir avanzando en identificar cuáles son las creencias y argumentos, las 'buenas razones', que sustentan las prácticas de los actores docentes involucrados; y confrontarlas con otras argumentaciones vigentes, en vistas de poder avanzar en la elaboración de comprensiones y posibles explicaciones que contribuyan a mejorar nuestra educación.

\section{BIBLIOGRAFÍA}

Bellël, C., Muñoz, G., Pérez, L.M., Raczynski, D. "Gestión que explica buenos resultados en SiMCE", Revista Educación del Ministerio de Educación de Chile (№313), 2004, pp. 51-68.

BOUDON, Raymond: Raisons bonnes raisons, Paris PUF, 2003. 
FUENTEALBA, Rodrigo: La inserción profesional de profesores principiantes de Enseñanza Media del área Humanista de la Región Metropolitana, Tesis de Doctorado, Programa de Doctorado, Facultad de Educación, Pontificia Universidad Católica de Chile, 2003.

GARANT, Celine: 'L'accompagnement dans l'initiation à la pratique d'enseignement: invitation ou frein à l'émancipation professionnelle?", Jeunes enseignants et insertion professionnelle, De Boeck, Belgique, 1999, pp. 85-112.

GARCíA HUIDOBRO, Juan Eduardo. (coordinador): La Reforma a la Educación Chilena, Barcelona, Editorial Popular, 1999.

GUZMÁN, María Angélica.: Interacción emergentes en el aula: una aproximación desde su configuración curricular. Tesis de Doctorado, presentada al Programa Doctorado en Ciencias de la Educación de la Facultad de Educación de la Pontificia Universidad Católica de Chile, 2002.

GONZÁLEZ, María Elena.: Creencias, atribuciones y autoeficacia en profesores de enseñanza básica municipalizada. Tesis de Doctorado, presentada al Programa Doctorado en Ciencias de la Educación de la Facultad de Educación de la Pontificia Universidad Católica de Chile, 1999.

IIPE-UNESCO: Formación docente inicial, Buenos Aires, Informes Periodísticos, 2001.

LATORRE, Marisol: : Saber Pedagógico en uso: análisis del saber actuante en las prácticas pedagógicas de profesores en ejercicio. Tesis de Doctorado en cotutela, presentada a la Pontificia Universidad Católica de Chile y la Universidad René Descartes-Paris V-Sorbonne, 2002.

LATORRE, Marisol.: "Aportes para el análisis de las racionalidades presentes en las prácticas pedagógicas", Revista Estudios Pedagógicos, Facultad de Filosofía y humanidades de la Universidad Austral de Chile (Número 30), 2004, pp. 75-91.

OECD: (2004) Informe sobre la Educación en América Latina.

PASMANIK, Diana: La praxis educativa en la enseñanza de la lengua materna: un análisis desde la interactividad en el aula, Tesis de Doctorado, presentada al Programa Doctorado en Ciencias de la Educación de la Facultad de Educación de la Pontificia Universidad Católica de Chile, 2001.

ReIMERS, Fernando.: "Las buenas maestras en América Latina", Revista Persona y Sociedad, Universidad Alberto Hurtado-ILADES, Chile, abril, 2003, pp. 33-47.

RIVKIN, S.G., HANUSHEK, E.A., KAIN, J.F.: Teachers, schools and academique achievement, en www.educarchile.cl, 31.07.2003.

SCHIEFELBEIN, Ernesto y Paulina: "Eficiencia en educación básica en Chile. Evaluación de resultados y propuestas de políticas", Revista Persona y Sociedad, Universidad Alberto Hurtado-ILADES, Chile, abril, 2003, pp. 149-163.

VAILLANT, Denise Formación de formadores. Estado de la práctica, PREAL, (doc. № 25), Diciembre, 2002.

VILLALTA, Marco: Prácticas pedagógicas del docente y cultura escolar en la interacción didáctica de sala de clase, Tesis de Doctorado, presentada al Programa Doctorado en Ciencias de la Educación de la Facultad de Educación de la Pontificia Universidad Católica de Chile, 2000.

TICKLE, L. (Teacher induction: the way ahead, London, Open University Press, 2000. 


\title{
Contactar
}

Revista lberoamericana de Educación

\author{
Principal OEI
}

